Research Article

\title{
Uniform Continuity of Fractal Interpolation Function
}

\author{
Xuezai Pan $\mathbb{D}^{1},{ }^{1}$ Minggang Wang $\mathbb{D},{ }^{2}$ and Xudong Shang ${ }^{1}$ \\ ${ }^{1}$ School of Mathematics, Nanjing Normal University Taizhou College, Taizhou 225300, China \\ ${ }^{2}$ School of Mathematics, Nanjing Normal University, Nanjing 210046, China \\ Correspondence should be addressed to Minggang Wang; magic821204@sina.com
}

Received 18 July 2020; Revised 24 September 2020; Accepted 16 October 2020; Published 4 November 2020

Academic Editor: Jixiang Yang

Copyright (c) 2020 Xuezai Pan et al. This is an open access article distributed under the Creative Commons Attribution License, which permits unrestricted use, distribution, and reproduction in any medium, provided the original work is properly cited.

In order to research analysis properties of fractal interpolation function generated by the iterated function system defined by affine transformation, the continuity of fractal interpolation function is proved by the continuous definition of function and the uniform continuity of fractal interpolation function is proved by the definition of uniform continuity and compactness theorem of sequence of numbers or finite covering theorem in this paper. The result shows that the fractal interpolation function is uniformly continuous in a closed interval which is from the abscissa of the first interpolation point to that of the last one.

\section{Introduction}

In 1960s, fractal geometry was regarded as a new interdiscipline firstly discovered by American mathematician Mandlebrot [1-5]. On the one hand, because most extremely irregular graphics in nature and very irregular social phenomenon are researched in the fractal geometry field, fractal geometry is called natural geometry. Therefore, fractal geometry is applied in almost all fields, such as mathematics, physics, chemistry, engineering, social science, and art [6-9]. On the other hand, for research on the natural properties of fractal itself, many methods used in researching fractals have been found by experts from 1960s to now, for example, fractal dimension method [10], multifractal spectrum method [11], adaptive fuzzy output-feedback method of nonlinear system [12], and nonlinear iterated method [13]. Especially, the fractal interpolation function method has been paid more and more attention by mathematicians. The theory of fractal interpolation function generated by the iterated function system defined by affine transformation was firstly proposed by Barnsley [14-16] and Massopust $[17,18]$. They found that any part of a fractal graphic is similar to the whole, so they used mathematical language to express the similar iterated process. That is to say, first, the iterated function system consisting of affine transformation is defined and it is proved that the iterated function system has a unique attractor that is the fixed point. Second, according to the theory of iterated function system and selfsimilar theory, complex fractal graphic, the graphic of fractal interpolation curve (refer with Figure 1), or fractal interpolation surface (refer with Figure 2) can be generated by computer program. Finally, the dimension theory and integrability of fractal interpolation function have been studied by Barnsley and Massopust.

Based on the research of fractal interpolation function above, the continuity and uniform continuity of the fractal interpolation function-generated iterated function systemdefined affine mapping are proved in the paper.

\section{Main Concepts and Lemmas}

Definition 1 (see $[19,20]$ ). Let $f$ be a function defined on interval $I$. If $\forall \bar{x} \in I$ and $\forall \varepsilon>0$, there is a $\delta>0$, so that for any $x \in I$ and $|x-\bar{x}|<\delta \Longrightarrow|f(x)-f(\bar{x})|<\varepsilon$, we call that $f$ is continuous on the point $\bar{x}$ and the $f$ is called continuous function on $I$.

Definition 2 (see $[19,20]$ ). Let $f$ be a function defined on interval $I$. If $\forall \varepsilon>0$ and $\exists \delta>0$, so that for any $x^{\prime}, x^{\prime \prime} \in I$, $\left|x^{\prime}-x^{\prime \prime}\right|<\delta \Longrightarrow\left|f\left(x^{\prime}\right)-f\left(x^{\prime \prime}\right)\right|<\varepsilon$, the $f$ is called uniformly continuous function on $I$. 


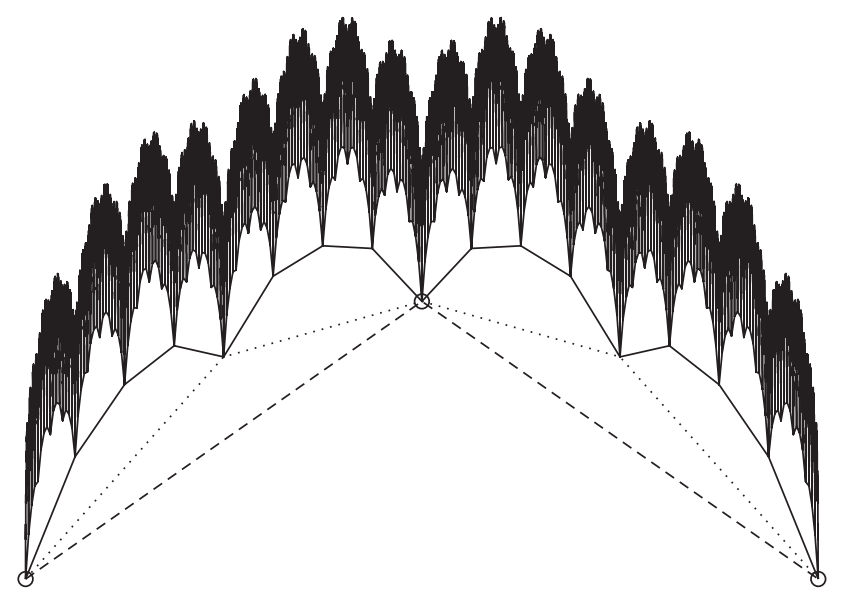

Figure 1: Fractal interpolation curve.

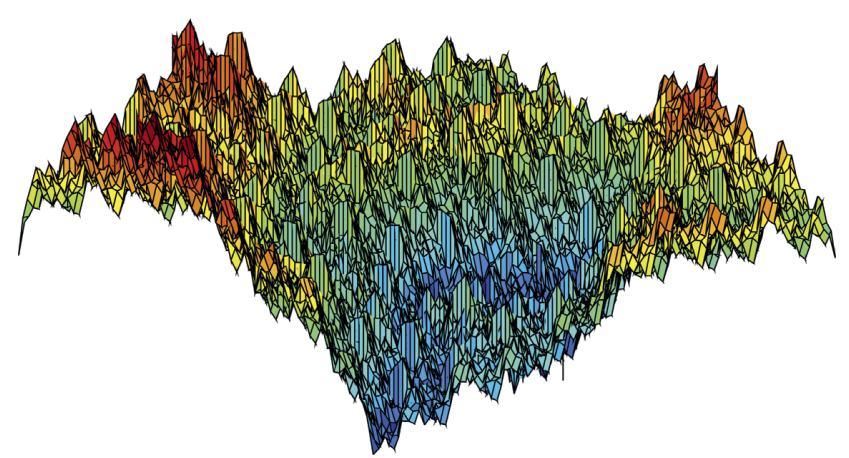

FIgURE 2: Fractal interpolation surface.

Definition 3 (see [19]). Let $S$ be a point subset on real number set $R$. Let $H$ be a open-interval set; in other words, each element in $H$ is the open-interval form of $(\alpha, \beta) . H$ is called an open covering of $S$ or $H$ covers $S$, if any point in $S$ is contained in at least an open interval in $H$. Furthermore, the number of open intervals in $H$ is infinite (or finite) and we call that $H$ is an infinite open covering of $S$ (or a finite open covering of $S$ ).

Definition 4 (see [14-16]). A (hyperbolic) iterated function system consists of a complete metric space $(X, d)$ together with a finite set of contraction mappings $w_{i}: X \longrightarrow X$, with respective contractivity mapping factors $s_{i}$, for $i=1,2, \ldots, n$. The abbreviation "IFS" is used for "iterated function system." The notation for the IFS just announced is $\left\{X ; w_{i}, i=1,2, \ldots, n\right\}$ and contractivity factor is $s=\max \left\{s_{i}: i=1,2, \ldots, n\right\}$.

Definition 5 (see [14]). Let $\left\{\left(x_{i}, y_{i}\right) \in R^{2} ; i=0,1,2, \ldots, n\right\}$ be a set of points, where $x_{0}<x_{1}<x_{2}<\cdots<x_{n}$. An interpolation function corresponding to this set of data is a function $f:\left[x_{0}, x_{n}\right] \longrightarrow R$ such that

$$
f\left(x_{i}\right)=y_{i}, \quad i=1,2, \ldots, n .
$$

The points $\left(x_{i}, y_{i}\right)$ are called the interpolation points. It is called that the function of $f$ interpolates the data and that the graph of $f$ passes through the interpolation points.

Lemma 1 (see $[19,20])$. If a sequence $\left\{x_{n}\right\}_{n=1}^{\infty}$ is bounded, the sequence $\left\{x_{n}\right\}$ has a convergent subsequence $\left\{x_{n_{k}}\right\}_{k=1}^{\infty}$.

Lemma 2 (see $[19,20]$ ). Let $H$ be a infinite open covering of closed interval $[a, b]$; then, there exist finite open intervals selected from $H$ to cover $[a, b]$.

Lemma 3 (see [14-18]). Let $n$ be a positive integer greater than 1. Let $\left\{R^{2} ; w_{i}, i=1,2, \ldots, n\right\}$ denote the IFS defined above, associated with the data set

$$
\left\{\left(x_{i}, y_{i}\right) \in R^{2} ; i=0,1,2, \ldots, n\right\} .
$$

Let the vertical scaling factor $d_{i}$ obey $0 \leq d_{i}<1$ for $i=1,2, \ldots, n$. Then, there is a metric $d$ on $R^{2}$, equivalent to the Euclidean metric, such that the IFS is hyperbolic with respect to $d$. In particular, there is a unique nonempty compact set $G \subset R^{2}$, such that

$$
G=\bigcup_{i=1}^{n} w_{i}(G) \text {. }
$$

In particular, an IFS of the form $\left\{R^{2} ; w_{i}, i=1,2, \ldots, n\right\}$ is considered, where the mapping is an affine transformation of the special structure

$$
w_{i}\left(\begin{array}{l}
x \\
y
\end{array}\right)=\left(\begin{array}{ll}
a_{i} & 0 \\
c_{i} & d_{i}
\end{array}\right)\left(\begin{array}{l}
x \\
y
\end{array}\right)+\left(\begin{array}{c}
e_{i} \\
f_{i}
\end{array}\right),
$$

The transformations are constrained by the data according to

$$
\begin{aligned}
& w_{i}\left(\begin{array}{c}
x_{0} \\
y_{0}
\end{array}\right)=\left(\begin{array}{c}
x_{i-1} \\
y_{i-1}
\end{array}\right), \\
& w_{i}\left(\begin{array}{c}
x_{n} \\
y_{n}
\end{array}\right)=\left(\begin{array}{c}
x_{i} \\
y_{i}
\end{array}\right), \quad \text { for } i=1,2, \ldots, n,
\end{aligned}
$$

$a_{i}, e_{i}, c_{i}$, and $f_{i}$ can be solved from equations (4)-(5) in terms of the data and vertical scaling factor $d_{i}$ :

$$
\begin{aligned}
& a_{i}=\frac{x_{i}-x_{i-1}}{x_{n}-x_{0}}, \\
& e_{i}=\frac{x_{n} x_{i-1}-x_{0} x_{i}}{x_{n}-x_{0}}, \\
& c_{i}=\frac{y_{i}-y_{i-1}}{x_{n}-x_{0}}-\frac{d_{i}\left(y_{n}-y_{0}\right)}{x_{n}-x_{0}}, \\
& f_{i}=\frac{x_{n} y_{i-1}-x_{0} y_{i}}{x_{n}-x_{0}}-\frac{d_{i}\left(x_{n} y_{0}-x_{0} y_{n}\right)}{x_{n}-x_{0}} .
\end{aligned}
$$

Lemma 4 (see [14-16]). Suppose $F$ is a set of continuous functions which satisfy $f:\left[x_{0}, x_{n}\right] \longrightarrow R$ and $f\left(x_{0}\right)=y_{0}$ 
and $f\left(x_{n}\right)=y_{n}$. The metric is defined by the following formula:

$$
d(f, g)=\max |f(x)-g(x)|, x \in\left[x_{0}, x_{n}\right], \quad \forall f, g \in F .
$$

Then, $(F, d)$ is a complete metric space. Let the real numbers $a_{i}, c_{i}, e_{i}$, and $f_{i}$, be defined by equations (5)-(9). Define a mapping $T: F \longrightarrow F$ by

$$
\begin{aligned}
(T f)(x)= & c_{i} L_{i}^{-1}(x)+d_{i} f\left(L_{i}^{-1}(x)\right) \\
& +f_{i}, x \in\left[x_{i-1}, x_{i}\right], \quad i=1,2, \ldots, n,
\end{aligned}
$$

where $L_{i}:\left[x_{0}, x_{n}\right] \longrightarrow\left[x_{i-1}, x_{i}\right]$ is the invertible transformation:

$$
\begin{aligned}
L_{i}(x) & =a_{i} x+e_{i}, \\
L_{i}^{-1}(x) & =\frac{x-e_{i}}{a_{i}}, L_{i}^{-1}\left(x_{i-1}\right)=x_{0}, L_{i}^{-1}\left(x_{i}\right) \\
& =x_{n} .
\end{aligned}
$$

$$
\begin{aligned}
\|f(x)-f(\bar{x})\| \| & =|T f(x)-T f(\bar{x})| \\
& =\left|c_{i} L_{i}^{-1}(x)+d_{i} f\left(L_{i}^{-1}(x)\right)+f_{i}-c_{i} L_{i}^{-1}(\bar{x})-d_{i} f\left(L_{i}^{-1}(\bar{x})\right)-f_{i}\right| \\
& =\mid c_{i}\left(\left(L_{i}^{-1}(x)-L_{i}^{-1}(\bar{x})\right)+d_{i}\left(f\left(L_{i}^{-1}(x)\right)-f\left(L_{i}^{-1}(\bar{x})\right)\right) \mid\right. \\
& =\left|c_{i}\left(\frac{x-e_{i}}{a_{i}}-\frac{\bar{x}-e_{i}}{a_{i}}\right)+d_{i}\left(f\left(\frac{x-e_{i}}{a_{i}}\right)-f\left(\frac{\bar{x}-e_{i}}{a_{i}}\right)\right)\right| \\
& =\mid \frac{c_{i}}{a_{i}}(x-\bar{x})+d_{i}\left(c_{i} x+d_{i}\left(\frac{x-e_{i}}{a_{i}}\right)+f_{i}-c_{i} \bar{x}-d_{i}\left(\frac{\bar{x}-e_{i}}{a_{i}}\right)-f_{i} \mid\right. \\
& =\left|\left(\frac{c_{i}+d_{i}^{2}}{a_{i}}+c_{i} d_{i}\right)(x-\bar{x})\right|<\varepsilon .
\end{aligned}
$$

Select

$$
p=\max _{1 \leq i \leq n}\left\{\left|\frac{c_{i}+d_{i}^{2}}{a_{i}}+c_{i} d_{i}\right|\right\} .
$$

Then, we select

$$
\delta=\frac{\varepsilon}{p}
$$

so $\forall \varepsilon>0$, for any $x \in\left[x_{0}, x_{n}\right]$ and $|x-\bar{x}|<\delta$, such that

$$
|f(x)-f(\bar{x})|<\varepsilon \text {. }
$$

Therefore, according to Definition 1, fractal interpolation function $f$ is continuous on the point $\bar{x}$. Furthermore, because $\bar{x}$ is arbitrarily selected from $\left[x_{0}, x_{n}\right]$ and $f$ is a continuous function on $\left[x_{0}, x_{n}\right]$.
Then, $T f$ is continuous on the interval $\left[x_{i-1}, x_{i}\right]$ and $T$ is a contraction mapping on $(F, d)$, so $T$ possesses a unique fixed point in $F$. That is, there exists a function $f \in F$ such that

$$
T f=f, \quad \forall f \in F .
$$

The function $f$ is called fractal interpolation function. The abbreviation "FIF" is used for "fractal interpolation function."

\section{The Continuity and the Uniform Continuity of Fractal Interpolation Function}

Theorem 1. Let function $f$ be a FIF generated by IFS mentioned in Definition 4 and defined by affine mapping referred from equations (3)-(14) and Lemmas 3 and 4. Then, the FIF is continuous on the closed interval on the closed interval $\left[x_{0}, x_{n}\right]$.

Proof. $\forall \bar{x} \in\left[x_{0}, x_{n}\right]$, according to Lemmas 3 and 4 and equations (11) to (14), $\forall \varepsilon>0$, and 
that is,

$$
\left|x_{m}^{\prime}-x_{m}^{\prime \prime}\right|<\delta
$$

but

$$
\left|f\left(x_{m}^{\prime}\right)-f\left(x_{m}^{\prime \prime}\right)\right| \geq \varepsilon_{0} .
$$

Because $x_{m}^{\prime} \in\left[x_{0}, x_{n}\right]$, the number sequence $\left\{x_{m}^{\prime}\right\}$ is a bounded number sequence. According to Lemma $1,\left\{x_{m}^{\prime}\right\}$ has a convergent subsequence $\left\{x_{m_{k}}^{\prime}\right\}$. Let $\left\{x_{m_{k}}^{\prime \prime}\right\} \longrightarrow \bar{x}$; then,

$$
\begin{aligned}
\lim _{k \longrightarrow \infty} x_{m_{k}}^{\prime \prime} & =\lim _{k \longrightarrow \infty}\left(x_{m_{k}}^{\prime \prime}-x_{m_{k}}^{\prime}+x_{m_{k}}^{\prime}\right) \\
& =\lim _{k \longrightarrow \infty} x_{m_{k}}^{x}=\bar{x},
\end{aligned}
$$

from $x_{0} \leq x_{m_{k}}^{\prime} \leq x_{n} \Longrightarrow x_{0} \leq \bar{x} \leq x_{n}$. Because FIF is a continuous function on the closed interval $\left[x_{0}, x_{n}\right]$,

$$
\lim _{x \longrightarrow \bar{x}} f(x)=f(\bar{x}) .
$$

From the relationship between limit of function and that of number sequence,

$$
\begin{aligned}
& \lim _{k \longrightarrow \infty}\left|f\left(x_{m_{k}}^{\prime}\right)-f\left(x_{m_{k}}^{\prime \prime}\right)\right| \geq \varepsilon_{0}, \\
& |f(\bar{x})-f(\bar{x})| \geq \varepsilon_{0},
\end{aligned}
$$

that is,

$$
0 \geq \varepsilon_{0},
$$

which is contrary to $\varepsilon_{0}>0$. In other words, at first, the contrary hypothesis to the conclusion of Theorem 2 makes $\varepsilon_{0}>0$ and $\varepsilon_{0} \leq 0$ contradictory. So, $f$ is uniform continuous function on the interval $\left[x_{0}, x_{n}\right]$.

For the proof of Theorem 2, we have the following second method to prove Theorem 2 .

Proof. Because fractal interpolation function $f$ is continuous on the interval $\left[x_{0}, x_{n}\right], \forall \bar{x} \in\left[x_{0}, x_{n}\right], \forall \varepsilon>0, \exists \delta_{\bar{x}}>0$, so that $\forall x \in \bigcup\left(\bar{x} ; t \delta_{\bar{x}}\right)$ and

$$
|f(x)-f(\bar{x})|<\varepsilon / 2 .
$$

Let

$$
H=\bigcup\left(\bar{x} ; t \frac{\delta_{\bar{x}}}{2}\right) \mid \bar{x} \in\left[x_{0}, x_{n}\right] .
$$

Obviously, $H$ is an open covering of $\left[x_{0}, x_{n}\right]$. According to Lemma 2 above, there exists

$$
H^{*}=\left\{\bigcup\left(\bar{x}_{i} ; \frac{\delta_{i}}{2}\right) \mid t n i q=h 1,2, x \ldots 7, C N\right\},
$$

to cover $\left[x_{0}, x_{n}\right]$. Suppose that

$$
\delta=\min _{1 \leq i \leq N}\left\{\frac{\delta_{i}}{2}\right\},
$$

$\forall x^{\prime}, x^{\prime \prime} \in\left[x_{0}, x_{n}\right]$, and $\left|x^{\prime}-x^{\prime \prime}\right|<\delta$; then, $\exists \bigcup\left(\bar{x}_{i} ; \delta_{i} / 2\right) \in$ $H^{*}$, such that

$$
x^{\prime} \in \bigcup\left(\bar{x}_{i} ; \frac{\delta_{i}}{2}\right) .
$$

On the one hand,

$$
\left|x^{\prime}-\bar{x}_{i}\right|<\frac{\delta_{i}}{2}<\delta_{i} \Longrightarrow\left|f\left(x^{\prime}\right)-f\left(\bar{x}_{i}\right)\right|<\frac{\varepsilon}{2} .
$$

On the other hand,

$$
\begin{aligned}
\left|x^{\prime \prime}-\bar{x}_{i}\right| & =\left|x^{\prime \prime}-x^{\prime}+x^{\prime}-\bar{x}_{i}\right|<\delta+\frac{\delta_{i}}{2}<\delta_{i} \\
& \Longrightarrow\left|f\left(x^{\prime \prime}\right)-f\left(\bar{x}_{i}\right)\right|<\frac{\varepsilon}{2} .
\end{aligned}
$$

Therefore,

$$
\begin{aligned}
\left|f\left(x^{\prime}\right)-f\left(x^{\prime \prime}\right)\right|= & \mid f\left(x^{\prime}\right)-f\left(\bar{x}_{i}\right) \\
& +f\left(\bar{x}_{i}\right) f\left(x^{\prime \prime}\right) \mid<\frac{\varepsilon}{2}+\frac{\varepsilon}{2}=\varepsilon .
\end{aligned}
$$

According to Definition 2, fractal interpolation function is a uniformly continuous function proved by finite covering Lemma 2.

In other words, from the two proof methods of Theorem 2 , fractal interpolation function $f$ is uniform continuous on the interval $\left[x_{0}, x_{n}\right]$.

\section{Conclusion}

From the above related theory of fractal interpolation function and function continuity, the following conclusion can be acquired through the above proof. Fractal interpolation function is not only continuous but also uniformly continuous on the closed interval $\left[x_{0}, x_{n}\right]$.

\section{Data Availability}

The data of this paper are available from the corresponding author upon request.

\section{Conflicts of Interest}

The authors declare that they have no conflicts of interest regarding the publication of this paper.

\section{Acknowledgments}

This research was supported by the overseas research program for outstanding young and middle-aged teachers and principals in Jiangsu Province. This research was funded by the following foundations: the National Natural Science Foundation of China (71503132 and 71811520710), Qing Lan Project of Jiangsu Province (2017), and Six Talent Peaks Project in Jiangsu Province (JY-055). This work was also supported by NSFC (11601234) and Natural Science Foundation of Jiangsu Province of China for Young Scholar (BK20160571). 


\section{References}

[1] B. Mandelbrot, "How long is the coast of Britain? statistical self-similarity and fractional dimension," Science, vol. 156, no. 3775, pp. 636-638, 1967.

[2] B. B. Mandelbrot and J. W. Van Ness, "Fractional brownian motions, fractional noises and applications," SIAM Review, vol. 10, no. 4, pp. 422-437, 1968.

[3] B. B. Mandelbrot, The Fractal Geometry of Nature, Macmillan Press, New York, NY, USA, 1983.

[4] B. B. Mandelbrot, D. E. Passoja, and A. J. Paullay, "Fractal character of fracture surfaces of metals," Nature, vol. 308, no. 5961, pp. 721-722, 1984.

[5] B. B. Mandelbrot, Fractal objects: Form ,opportunity, and Dimension, Peking, Beijing World Publishing Corporation, Beijing, China, 1999.

[6] H. Xie, Fractals in Rock Mechanics, A. Balkerma Publisher, Rotterdam, Netherlands, 1993.

[7] H. P. Xie, H. Q. Sun, and Y. Ju, "Study on generation of rock surfaces by using fractal Interpolation," International Journal of Solid and Structure, vol. 38, no. 32-33, pp. 5765-5787, 2001.

[8] M. Nasehnejad, G. Nabiyouni, and M. Gholipour Shahraki, "Fractal analysis of nanostructured silver film surface," Chinese Journal of Physics, vol. 55, no. 6, pp. 2484-2490, 2017.

[9] S. Bianchi and M. Frezza, "Fractal stock markets: international evidence of dynamical (in) efficiency," Chaos: An Interdisciplinary Journal of Nonlinear Science, vol. 27, no. 7, Article ID 071102, 2017.

[10] Z. Feng, "Variation and minkowski dimension of fractal interpolation surface," Journal of Mathematical Analysis and Applications, vol. 345, no. 1, pp. 322-334, 2008.

[11] X. Pan, X. Shang, M. Wang, and F. Zuo-Fei, "The cantor set's multi-fractal spectrum formed by different probability factors in mathematical experiment," Fractals, vol. 25, no. 1, 8 pages, Article ID 1750002, 2017.

[12] Y. Chang, Y. Wang, F. E. Alsaadi, and G. Zong, "Adaptive fuzzy output-feedback tracking control for switched stochastic pure-feedback nonlinear systems," International Journal of Adaptive Control and Signal Processing, vol. 33, no. 10, pp. 1567-1582, 2019.

[13] B. Hong and D. Lu, "Modified fractional variational iteration method for solving the generalized time-space fractional schrödinger equation," The Scientific World Journal, vol. 2014, Article ID 964643, 1 page, 2014.

[14] M. F. Barnsley, "Lecture notes on iterated function systems," Proceedings of Symposia in Applied Mathematics, vol. 39, pp. 127-144, 1989.

[15] M. F. Barnsley, "Fractal functions and interpolation," Constructive Approximation, vol. 2, no. 1, pp. 303-329, 1986.

[16] M. F. Barnsley, Fractals Everywhere, 2nd edition, 2009, https:// www.google.com/search?rlz=1C1GCEU_enIN894IN894\&q = Amsterdam\&stick=H4sIAAAAAAAAAOPgE-LUz9U3MDJL SYpXYgcxs40LtLSyk63084vSE_MyqxJLMvPzUDhWGamJKYW liUUlqUXFilg5HXOLgayUxNwdrIwA9iXLVlEAAAA\&sa=X\&v ed=2ahUKEwiCuOaEucfsAhWW73MBHdUbDXQQmxMoAT AXegQIGhAD, 2nd edition.

[17] P. R. Massopust, "Fractal surfaces," Journal of Mathematical Analysis and Applications, vol. 151, no. 1, pp. 275-290, 1990.

[18] P. R. Massopust, Fractal Functions, Fractal Surfaces, and Wavelets, Academic Press, Orlando, FL, USA, 1995.

[19] School of Mathematical Sciences, East China Normal University, Mathematical Analysis, Higher Education Press, Beijing, China, (in Chinese), 2010.

[20] S. L. Xu and C. H. Xue, Mathematical Analysis, TsingHua University Press, Beijing, China, 2005. 